\title{
Probing the trilinear Higgs boson self-coupling via single Higgs production at the $\mathrm{LHeC}$
}

\author{
Ruibo Li®, ${ }^{*}$ Xiao-Min Shen $\odot,{ }^{\dagger}$ Bo-Wen Wang, ${ }^{\ddagger}$ Kai Wang, ${ }^{\S}$ and Guohuai Zhu® \\ Zhejiang Institute of Modern Physics, Department of Physics, Zhejiang University, \\ Hangzhou, Zhejiang 310027, China
}

(Received 6 November 2019; accepted 2 April 2020; published 20 April 2020)

\begin{abstract}
The determination of the Higgs self-coupling is one of the key ingredients for understanding the mechanism behind the electroweak symmetry breaking. An indirect method for constraining the Higgs trilinear self-coupling via single Higgs production at next-to-leading order (NLO) has been proposed in order to avoid the drawbacks of studies with double Higgs production. In this paper we study the Higgs self-interaction through the vector boson fusion (VBF) process $e^{-} p \rightarrow \nu_{e} h j$ at the future LHeC. At NLO level, we compute analytically the scattering amplitudes for relevant processes, in particular those induced by the Higgs self-interaction. A Monte Carlo simulation and a statistical analysis utilizing the analytic results are then carried out for Higgs production through VBF and decay to $b \bar{b}$, which yield for the trilinear Higgs self-coupling rescaling parameter $\kappa_{\lambda}$ the limit $[-0.57,2.98]$ with $2 \mathrm{ab}^{-1}$ integrated luminosity. If we assume about $10 \%$ of the signal survives the event selection cuts, and include all the background, the constraint will be broadened to $[-2.11,4.63]$.
\end{abstract}

DOI: 10.1103/PhysRevD.101.075036

\section{INTRODUCTION}

A standard model (SM)-like Higgs boson has been discovered by the ATLAS and CMS collaborations at the CERN Large Hadron Collider (LHC) individually [1,2], which makes a milestone in particle physics. While it strongly supports the SM mechanism of spontaneous electroweak symmetry breaking (EWSB), by which all fermions and some of the vector bosons acquire their masses, the driving force of EWSB still remains mysterious. To better understand this problem, it is crucial to study the properties of the Higgs boson, e.g., to measure its mass, spin, $C P$ properties and couplings [3-6]. From the second run of the LHC at $13 \mathrm{TeV}$, the ATLAS collaboration has recently reported the results of their measurements $\mu_{H \rightarrow \tau \tau}=$ $1.09_{-0.30}^{+0.36}$ and $\mu_{H \rightarrow b b}=1.01_{-0.19}^{+0.20}$, with the integrated luminosities $36.1 \mathrm{fb}^{-1}$ and $79.8 \mathrm{fb}^{-1}$, respectively [7,8]. These are significant improvements in Higgs precision physics.

\footnotetext{
*bobli@zju.edu.cn

†xmshen@zju.edu.cn

*0617626@zju.edu.cn

\$wangkai1@zju.edu.cn

"zhugh@zju.edu.cn
}

Published by the American Physical Society under the terms of the Creative Commons Attribution 4.0 International license. Further distribution of this work must maintain attribution to the author(s) and the published article's title, journal citation, and DOI. Funded by SCOAP ${ }^{3}$.
Equally important is the measurement of the Higgs selfcoupling $(\lambda)$ from the scalar potential $V(\Phi)$, which takes a form with the trilinear $\left(\lambda_{3}^{\mathrm{SM}}=\lambda\right)$ and quartic $\left(\lambda_{4}^{\mathrm{SM}}=\lambda / 4\right)$ self-interactions:

$V(\Phi)=-\mu^{2} \Phi^{\dagger} \Phi+\lambda\left(\Phi^{\dagger} \Phi\right)^{2} \rightarrow \frac{1}{2} m_{h}^{2} h^{2}+\lambda_{3} \nu h^{3}+\lambda_{4} h^{4}$,

where $\Phi$ is the Higgs doublet field and $h$ is the Higgs boson. In addition to its crucial role in EWSB, the value of $\lambda$ has interesting implications on physics beyond the SM (BSM). For example, in electroweak baryogenesis, a large deviation of $\lambda$ from its SM value has been used to explain the observed cosmic baryon-antibaryon asymmetry [9-11].

In contrast to the measurement of Higgs-fermion couplings, the study of $\lambda$ is in a completely different situation. At the LHC, double Higgs production as the standard process for determining the Higgs trilinear self-coupling suffers from a small production rate and huge QCD backgrounds, and thus leads to large uncertainties even after the Run-II upgrade. The measurements of the $\gamma \gamma b \bar{b}$ final states by the CMS and ATLAS experiments yield the constraints $-11 \lambda_{3}^{\mathrm{SM}}<\lambda_{3}<17 \lambda_{3}^{\mathrm{SM}}$ and $-8.2 \lambda_{3}^{\mathrm{SM}}<\lambda_{3}<$ $13.2 \lambda_{3}^{\mathrm{SM}}$, respectively $[12,13]$. For the $b \bar{b} b \bar{b}$ production, the observed upper limit by ATLAS using the nonresonant Higgs pair production data is 13 times the SM value at 95\% C.L. [14]. Combining the measurements of the different final states, CMS and ATLAS report their limits $-11.8 \lambda_{3}^{\mathrm{SM}}<\lambda_{3}<18.8 \lambda_{3}^{\mathrm{SM}}$ and $-5.0 \lambda_{3}^{\mathrm{SM}}<\lambda_{3}<12.0 \lambda_{3}^{\mathrm{SM}}$ 
$[15,16]$. There are also extensive phenomenological studies on determining the trilinear Higgs self-coupling directly at the LHC (including the prospect studies for the high-energy and high-luminosity upgrades of the LHC) [17-30], the future electron-positron collider [31-34], and future high energy hadron colliders [35-46], in which strict constraints are obtained with higher integrated luminosities and energies. On the other hand, an indirect method is proposed for constraining the Higgs self-coupling via single Higgs production at next-to-leading order (NLO) [33,47-51]. The method relies on the account of one-loop electroweak radiative corrections to Higgs-strahlung and vector boson fusion (VBF) processes [52-55], and it has the potential of reaching a superior precision in the determination of the Higgs self-coupling. From the single Higgs production measurement, ATLAS obtained recently the constraint $-3.2 \lambda_{3}^{\mathrm{SM}}<\lambda_{3}<11.9 \lambda_{3}^{\mathrm{SM}}[56]$. The combination of double and single Higgs production measurements gives $-2.3 \lambda_{3}^{\mathrm{SM}}<$ $\lambda_{3}<10.3 \lambda_{3}^{\mathrm{SM}}[57]$.

In view of the large QCD backgrounds interfering with the one-loop electroweak radiative corrections at the hadron-hadron collider, the Large Hadron electron Collider ( $\mathrm{LHeC}$ ) has been proposed as a deep inelastic scattering facility for the precision measurement of parton distributions and Higgs properties. $\mathrm{LHeC}$ as a relatively economic proposal is an upgrade based on the current $7 \mathrm{TeV}$ proton beam of the LHC by adding one electron beam with 60-140 GeV energy [58], which could be tuned into a "Higgs factory" in which Higgs bosons are produced via VBF process. Thanks to the forward detector and reduction of QCD backgrounds in the $e-p$ collider, the bottom Yukawa and trilinear Higgs self-couplings could be measured precisely [59-61]. Therefore, we expect the $\mathrm{LHeC}$ to be a good facility for studying $\lambda_{3}$ via single Higgs production at NLO level.

One may try to constrain $\lambda_{3}$ at the LHeC via Higgs pair production. The process has been studied in Ref. [60]. Combining the pair production cross section at the $\mathrm{LHeC}$ with the signal and background selection efficiencies from the work above, ${ }^{1}$ one can estimate that in order to obtain a $2 \sigma$ signal significance, the integrated luminosity needed would be about $23.6 \mathrm{ab}^{-1}$, much higher than the planned $2 \mathrm{ab}^{-1}$ at the LHeC. Hence we only focus on the study of single Higgs production hereafter.

The paper is organized as follows. In the next section, we discuss the one-loop contribution to single Higgs production, in particular that from processes via the trilinear Higgs self-interaction and Higgs top quark Yukawa interaction, and calculate their scattering amplitudes analytically. In Sec. III, we perform a Monte Carlo simulation for single

\footnotetext{
${ }^{1}$ Here we assume no significant change in the selection efficiencies at the LHeC, as compared to those in Ref. [60]. The full analysis of Higgs pair production is out of the scope of this study.
}

Higgs production at the $\mathrm{LHeC}$, produce the differential and total cross section, and carry out a statistical analysis to obtain constraints for $\lambda_{3}$. Finally, we conclude in Sec. IV.

\section{THE ONE LOOP CORRECTION TO SINGLE HIGGS PRODUCTION AT THE LHEC}

Given the tiny cross section of di-Higgs production [60], one could instead constrain the trilinear Higgs self-coupling $\lambda_{3}$ at the LHeC via the $\lambda_{3}$ induced loop corrections to the tree level single Higgs production process $e^{-} p \rightarrow \nu_{e} h j$ shown in Fig. 1. We parametrize the deviation of possible new physics from SM by a single parameter $\kappa_{\lambda}$ :

$$
\lambda_{3}^{\mathrm{SM}} h^{3} \rightarrow \lambda_{3} h^{3}=\kappa_{\lambda} \lambda_{3}^{\mathrm{SM}} h^{3},
$$

where the physical Higgs field $h$ has a zero vacuum expectation value $(\mathrm{VEV})$, and $\lambda_{3}^{\mathrm{SM}} \approx 0.13$ is the Higgs trilinear selfcoupling in the SM. Other parameters are assumed to be fixed at their SM values. Realistic scenarios have been discussed in [47]. For example, the $\left(|\Phi|^{2}-v^{2} / 2\right)^{3} / \Lambda^{2}$ operator, with appropriately tuned parameters, leads to deviation of the $h^{3}$ coupling, leaving the Higgs mass term, VEV, and other trilinear couplings (such as the couplings of Higgs and Goldstone $h G^{+} G^{-}, h G^{0} G^{0}$ ) unchanged. ${ }^{2}$ Another example is the $C P$-conserving two Higgs doublet model (2HDM). In the decoupling limit of $2 \mathrm{HDM}$, one has $\delta \kappa_{\lambda} / \delta \kappa_{h V V} \simeq$ $4 m_{A}^{2} / m_{h}^{2} \gg 1$ [62], where $\delta \kappa_{h V V}$ is the relative deviation of $H W W, H Z Z$ coupling, $m_{A}$ is the mass of the $C P$-odd scalar, $m_{h}$ is the mass of the lighter $C P$-even scalar. For large $m_{A}$, the loop-level modification due to $\kappa_{\lambda}$ may overwhelm the tree-level deviation due to the anomalous $H V V$ couplings [47], and the above simplified model provides a good approximation.

In the following, we shall identify various contributions up to NLO that are relevant for constraining the Higgs trilinear coupling.

\section{A. Trilinear Higgs self-coupling: $\lambda_{3}$}

In this section we consider only the production of Higgs boson. Note, however, the decay of Higgs boson is also $\lambda_{3}$ dependent, and has been studied in [49].

For VBF process at the $\mathrm{LHeC}$, the trilinear Higgs selfcoupling $\lambda_{3}$ enters at one-loop level. The $\lambda_{3}$-dependent oneloop corrections to the $H W W$ vertex are calculated in the unitary gauge. The relevant Feynman diagrams are shown in Fig. 2. To check the correctness and gauge invariance of our result, we compute the same process in the $R_{\xi}$ gauge

\footnotetext{
${ }^{2}$ This operator, after electroweak symmetry breaking, also leads to deviation of operators with dimension $D \geq 4$, such as $h^{4}$, $h^{4} G^{+} G^{-}$. Our study of the trilinear Higgs coupling (calculation of loop diagrams, expressions of renormalization constants, wave function corrections), however, will not be spoiled by the possible deviation of these marginal or irrelevant operators.
} 


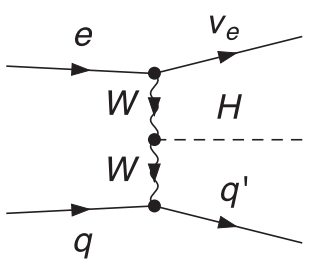

FIG. 1. The Feynman diagram of single Higgs production via $W$ boson fusion at leading order with $q=u, c, \bar{d}, \bar{s}$ and $q^{\prime}=$ $d, s, \bar{u}, \bar{c}$ at the $\mathrm{LHeC}$.

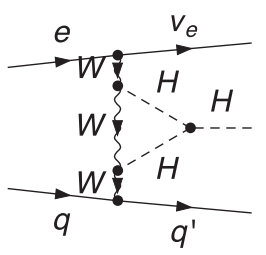

(a)

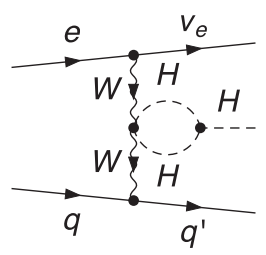

(b)

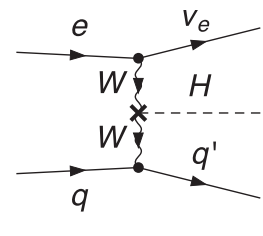

(c)
FIG. 2. The $\lambda_{3}$-dependent Feynman diagrams and the corresponding counterterm in unitary gauge at one-loop level with $q=u, c, \bar{d}, \bar{s}$ and $q^{\prime}=d, s, \bar{u}, \bar{c}$.

(where an additional diagram with Goldstone boson is needed) with the FEYNARTS and FORMCALC packages $[63,64]$. The result is independent of $\xi$ and equals to our result obtained in the unitary gauge.

The $\lambda_{3}$-dependence of Higgs wave function correction comes from the bubble diagram and the counterterm diagram, as shown in Fig. 3.

As to the renormalization of the theory, we follow the framework given in Ref. [65] and take $\kappa_{\lambda}, e, M_{H}, M_{W}, M_{Z}$ as input parameters. Note that unlike Ref. [65], which concerns the renormalization of the Standard Model, we introduce an additional input parameter $\kappa_{\lambda}$. Scrutiny of Ref. [65] shows that most of the results there are still valid in our model. Major difference appears in the wave function renormalization constant of Higgs boson, denoted by $\delta Z_{H}$. As we will see in Sec. II C, the contribution of $\delta Z_{H}$ to the $H W W$ vertex counter term cancels its contribution to the counterterm of Higgs wave function correction. The Higgs field $h$ is constrained by renormalization condition to have a zero VEV.

Combining Figs. 2 and 3, we obtain the $\lambda_{3}$-dependent one-loop contribution to the amplitude of VBF process. The explicit expressions will be given in Sec. II C.

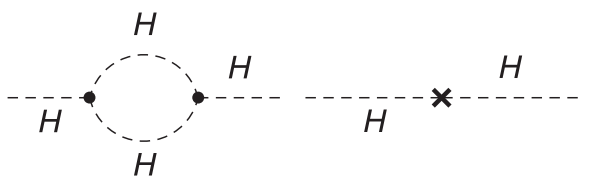

(a)

(b)

FIG. 3. $\lambda_{3}$-dependent corrections to the Higgs wave function.

\section{B. Top quark Yukawa coupling: $y_{t}$}

The same final states can be produced through the top quark Yukawa coupling to the Higgs boson. Because of the large coupling strength $y_{t}$ and the insertion $M_{t}$ in the loop, the contribution from this channel may be sizable and could affect the determination of the trilinear Higgs self-coupling. Therefore, we shall treat it as an irreducible background and compute its contribution. As it is not straightforward to separate the contribution from top and bottom quarks in the renormalization constants, we calculate all the contribution of top and bottom quarks by taking $\kappa_{\lambda}, e, M_{H}, M_{W}, M_{Z}$, and $M_{t}$ as input parameters in the $M_{b} \rightarrow 0$ limit. The Feynman diagrams in the unitary gauge are shown in Fig. 4. (Diagrams that vanish in the $M_{b} \rightarrow 0$ limit are not shown here.) The full analytic result will be shown in Sec. II C.

\section{Analytical result}

In this section, we give the analytical result in both onshell and $\overline{\mathrm{MS}}$ schemes. We shall use $\overline{\mathrm{MS}}$ scheme in our numerical simulation in the next section.

The momenta of the electron, incoming parton, Higgs boson, electron neutrino, and outgoing parton are denoted by $p_{1}, p_{2}, \ldots, p_{5}$ respectively. The Mandelstam variables are defined as $S_{i j} \equiv\left(p_{i}+p_{j}\right)^{2}, T_{i j} \equiv\left(p_{i}-p_{j}\right)^{2}$. In this work the masses of $u, c, d, s$ quarks are neglected. The CKM matrix elements $V_{u b}, V_{c b}, V_{t d}, V_{t s}$ are also taken to be zero.

We expand the amplitude $\mathcal{M}_{q}$ of our process in powers of $g_{W} \equiv \sqrt{\frac{4 \pi \alpha_{e}}{\sin ^{2} \theta_{W}}}$ as

$$
\mathcal{M}_{q}=g_{W}^{3} \mathcal{M}_{q}^{(0)}+g_{W}^{5} \mathcal{M}_{q}^{(1)}+\cdots
$$

where $q$ stands for the incoming parton, $\alpha_{e}$ is the fine structure constant and $\theta_{W} \equiv \arccos \frac{M_{W}}{M_{Z}}$ is the Weinberg angle. The squared amplitude is then given by

$$
\begin{aligned}
\frac{1}{2} \sum_{s} \frac{1}{3} \sum_{\text {color }}\left|\mathcal{M}_{q_{s}, e_{L}}\right|^{2}= & \frac{1}{8} g_{W}^{6} M_{W}^{2} \frac{1}{\left(T_{14}-M_{W}^{2}\right)^{2}\left(T_{25}-M_{W}^{2}\right)^{2}} \mathcal{F}_{q}^{(1)}+\frac{3 M_{H}^{2}}{128 \pi^{2}} g_{W}^{8} \frac{1}{\left(T_{14}-M_{W}^{2}\right)^{2}\left(T_{25}-M_{W}^{2}\right)^{2}} \times G_{\lambda} \\
& -\frac{N_{c} M_{t}^{2}}{128 \pi^{2}} g_{W}^{8} \frac{1}{\left(T_{14}-M_{W}^{2}\right)^{2}\left(T_{25}-M_{W}^{2}\right)^{2}} \times G_{t+b}^{(3)} \\
& +\left[\frac{N_{c} M_{W}^{2}}{128 \pi^{2}} g_{W}^{8} \frac{1}{\left(T_{14}-M_{W}^{2}\right)^{3}\left(T_{25}-M_{W}^{2}\right)^{2}} \times G_{t+b}^{(14)}+14 \leftrightarrow 25\right]+\cdots,
\end{aligned}
$$




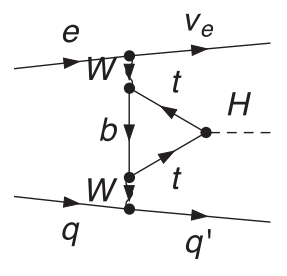

(a)

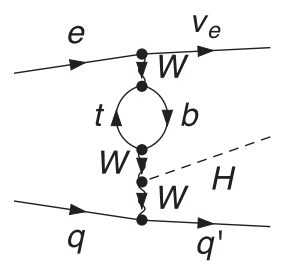

(c)

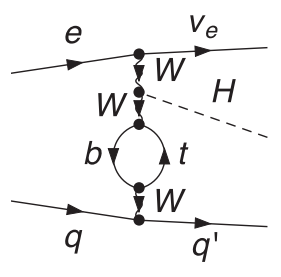

(d)

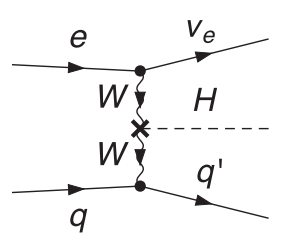

(b)

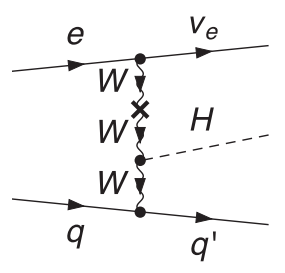

(e)

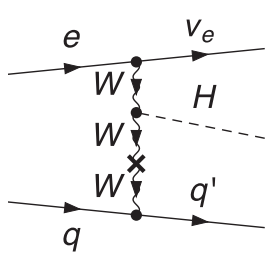

(f)

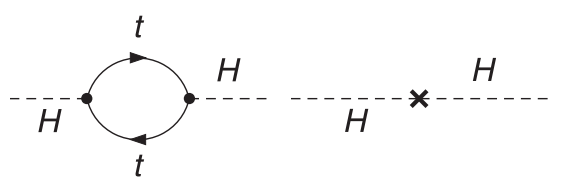

(g)

(h)

FIG. 4. Feynman diagrams with top and bottom loops and the corresponding counterterms in the unitary gauge at one loop level with $q=u, c, \bar{d}, \bar{s}$ and $q^{\prime}=d, s, \bar{u}, \bar{c}$.

where $s$ is spin of the incoming quark(antiquark), the subscripts of $\mathcal{M}$ denote the flavor and spin of the incoming quark(antiquark) and electron. The polarization of the electron beam will be considered in next section. The fermion chains $\mathcal{F}_{q}^{(1)}$ are

$$
\begin{aligned}
\mathcal{F}_{u, c}^{(1)}= & 4 S_{12} S_{45} \\
\mathcal{F}_{\bar{d}, \bar{s}}^{(1)}= & 4 T_{15} T_{24} \\
\mathcal{F}_{u, c}^{(2)}= & \left(S_{12}+S_{45}\right)\left(S_{12} S_{45}+T_{15} T_{24}-T_{14} T_{25}\right) \\
& +2 S_{12} S_{45}\left(T_{15}+T_{24}\right) \\
\mathcal{F}_{\bar{d}, \bar{s}}^{(2)}= & \left(T_{15}+T_{24}\right)\left(S_{12} S_{45}+T_{15} T_{24}-T_{14} T_{25}\right) \\
& +2 T_{15} T_{24}\left(S_{12}+S_{45}\right),
\end{aligned}
$$

where $q=u, c, \bar{d}, \bar{s}$ is the incoming parton. The $\kappa_{\lambda}$ dependent term $G_{\lambda}$ reads

$$
\begin{aligned}
G_{\lambda}= & {\left[\left(C_{00}-\frac{1}{4} B_{0}-M_{W}^{2} C_{0}\right) \mathcal{F}_{q}^{(1)}-C_{12} \mathcal{F}_{q}^{(2)}\right] \kappa_{\lambda} } \\
& -\frac{3}{8} \kappa_{\lambda}^{2} M_{H}^{2} B_{0}^{\prime} \mathcal{F}_{q}^{(1)} .
\end{aligned}
$$

Note that the contribution from $\kappa_{\lambda}$ dependent counterterms vanishes. $G_{\lambda}$ takes the same form in both OS and $\overline{\mathrm{MS}}$ schemes, but the quantities on which it depends are generally renormalization scheme dependent. The contribution from top and bottom quarks, in the $M_{b} \rightarrow 0$ limit, is given by

$$
\begin{aligned}
G_{t+b}^{(3)}= & \left\{4 C_{00}^{(t)}-2 B_{0}\left(T_{25}, M_{b}^{2}, M_{t}^{2}\right)-2\left(M_{t}^{2}-T_{14}\right) C_{0}^{(t)}\right. \\
& +\frac{1}{2}\left(M_{H}^{2}+5 T_{14}-T_{25}\right) C_{1}^{(t)} \\
& +\frac{1}{2}\left(-3 M_{H}^{2}+3 T_{14}+T_{25}\right) C_{2}^{(t)} \\
& \left.-\frac{1}{2}\left[\left(4 M_{t}^{2}-M_{H}^{2}\right) B_{0}^{(t)}-B_{0}^{(t)}\right]+\mathrm{CT}_{t+b}^{(3)}\right\} \mathcal{F}_{q}^{(1)} \\
& -\left(C_{1}^{(t)}+C_{2}^{(t)}+4 C_{12}^{(t)}\right) \mathcal{F}_{q}^{(2)} \\
G_{t+b}^{(14)}= & {\left[-4 B_{00}\left(T_{14}, M_{b}^{2}, M_{t}^{2}\right)+2\left(M_{t}^{2}-T_{14}\right) B_{0}\left(T_{14}, M_{b}^{2}, M_{t}^{2}\right)\right.} \\
& \left.-2 T_{14} B_{1}\left(T_{14}, M_{b}^{2}, M_{t}^{2}\right)+\mathrm{CT}_{t+b}^{(14)}\right] \mathcal{F}_{q}^{(1)}
\end{aligned}
$$

with contributions from counterterms [65] 


$$
\begin{aligned}
&\left.\mathrm{CT}_{t+b}^{(3)}\right|_{\mathrm{OS}}= 2 \frac{-16 \pi^{2} M_{W}^{2}}{N_{c} g_{W}^{2}} \frac{M_{t}^{2}}{} \\
& \times\left[\left(\delta Z_{e}-\frac{\delta s}{s}+\frac{1}{2} \frac{\delta M_{W}^{2}}{M_{W}^{2}}+\frac{1}{2} \delta Z_{H}+\delta Z_{W}\right)-\frac{1}{2} \delta Z_{H}\right]_{t+b} \\
&\left.\mathrm{CT}_{t+b}^{(3)}\right|_{\overline{\mathrm{MS}}}= \frac{1}{2}\left(\frac{2}{4-D}\right) \\
&\left.\mathrm{CT}_{t+b}^{(14)}\right|_{\mathrm{OS}}= \frac{32 \pi^{2}}{N_{C} g_{W}^{2}}(-1)\left[\delta Z_{W}\left(T_{14}-M_{W}^{2}\right)-\delta M_{W}^{2}\right]_{t+b} \\
&\left.\mathrm{CT}_{t+b}^{(14)}\right|_{\overline{\mathrm{MS}}}=\left(\frac{2}{3} T_{14}-M_{t}^{2}\right)\left(\frac{2}{4-D}\right)
\end{aligned}
$$

where the subscript $t+b$ represents the contribution from the top and bottom quarks, $D$ is the dimension of spacetime and $\delta Z_{e}, \delta Z_{H}, \delta Z_{W}, \delta s, \delta M_{W}^{2}$ are renormalization constants in the on-shell scheme. Detailed expressions of these renormalization constants can be found in [65] (Note that, at one loop level, all the renormalization constants in the above equations are independent of $\kappa_{\lambda}$ except for $\delta Z_{H}$, whose contribution cancels out in the final result.)

$B_{0}, B_{0}^{\prime}, C_{x}$ (e.g., $C_{00}, C_{1}$, etc.) are scalar integrals

$$
\begin{aligned}
C_{x} & =C_{x}\left(T_{14}, M_{H}^{2}, T_{25}, M_{W}^{2}, M_{H}^{2}, M_{H}^{2}\right) \\
B_{0} & =B_{0}\left(M_{H}^{2}, M_{H}^{2}, M_{H}^{2}\right) \\
B_{0}^{\prime} & =\left.\frac{\partial}{\partial s} B_{0}\left(s, M_{H}^{2}, M_{H}^{2}\right)\right|_{s=M_{H, \mathrm{ph}}^{2}}
\end{aligned}
$$

and

$$
\begin{aligned}
C_{x}^{(t)} & =C_{x}\left(T_{14}, M_{H}^{2}, T_{25}, M_{b}^{2}, M_{t}^{2}, M_{t}^{2}\right) \\
B_{0}^{(t)} & =B_{0}\left(M_{H}^{2}, M_{t}^{2}, M_{t}^{2}\right) \\
B_{0}^{\prime(t)} & =\left.\frac{\partial}{\partial s} B_{0}\left(s, M_{t}^{2}, M_{t}^{2}\right)\right|_{s=M_{H, \mathrm{ph}}^{2} .}
\end{aligned}
$$

Here $M_{H, \mathrm{ph}}$ is the physical mass of Higgs boson while values of other mass parameters, e.g., $M_{H}$, depend on the renormalization scheme used.

As a cross check, we repeat the calculation with FEYNCALC $[66,67]$ and obtain the same result. Gauge invariance is verified by working in the general $R_{\xi}$ gauge and making sure that no gauge parameter dependence remains in the final result.

\section{MONTE CARLO SIMULATION}

The squared amplitude in Eq. (4) can be turned into the NLO cross section $\sigma_{\kappa_{\lambda}}$ for the process $e^{-} p \rightarrow \nu_{e} h j$ after integration over the phase space of the final states and convolution with parton distribution function (PDF) of the incoming quark(antiquark). We use the Vegas algorithm implemented in the Cuba library [68] to perform the numerical integration in our simulation at parton level.
We also promote it to a parton level event generator to study the variation of kinematical variables. Given the numerical values of the input parameters, the scalar integrals $B_{0}, B_{0}^{\prime}$, $C_{x}$ (e.g., $C_{00}, C_{1}$, etc.) are evaluated with the LOOPTOOLS [64] package. The PDF set CT14qed_inc_proton is used [69]. Both the renormalization and factorization scales are set to $M_{H}=125 \mathrm{GeV}$. The following basic cuts are adopted:

$$
\begin{aligned}
p_{T}^{j} & >20 \mathrm{GeV}, \quad p_{T}^{\ell}>5 \mathrm{GeV} \\
\left|\eta_{j}\right| & <5, \quad\left|\eta_{\ell}\right|<5 \\
\mathscr{E}_{T} & >10 \mathrm{GeV} .
\end{aligned}
$$

With beam energies being $7 \mathrm{TeV}$ and $60 \mathrm{GeV}$ for the proton and electron, and electron polarization assumed to be $-80 \%$, the cross section of the process $e^{-} p \rightarrow \nu_{e} h j$ is $145 \mathrm{fb}$ at leading order. In Fig. 5, we show the cross section $\delta \sigma_{\kappa_{\lambda}}$ from only the $\kappa_{\lambda}$ dependent one loop terms. The quadratic form can be traced back to the $\kappa_{\lambda}$ and $\left(\kappa_{\lambda}\right)^{2}$ terms in Eq. (6). The universal contribution from the top and bottom quarks turns out to be $-1.8 \mathrm{fb}$. The Vegas phase space integration is cross checked with MadGraph5_V2.6.5 [70], which gives the consistent result.

One way to show the significance of $\kappa_{\lambda}$ is via the differential distributions of characteristic kinematic variables, such as the azimuthal angle $\Delta \phi_{\not_{T}^{\prime} j}$, the Higgs transverse momentum $p_{T}^{h}$, etc. Unfortunately, the discrimination between distributions for various processes relies heavily on the effect of threshold Sommerfeld enhancement, which

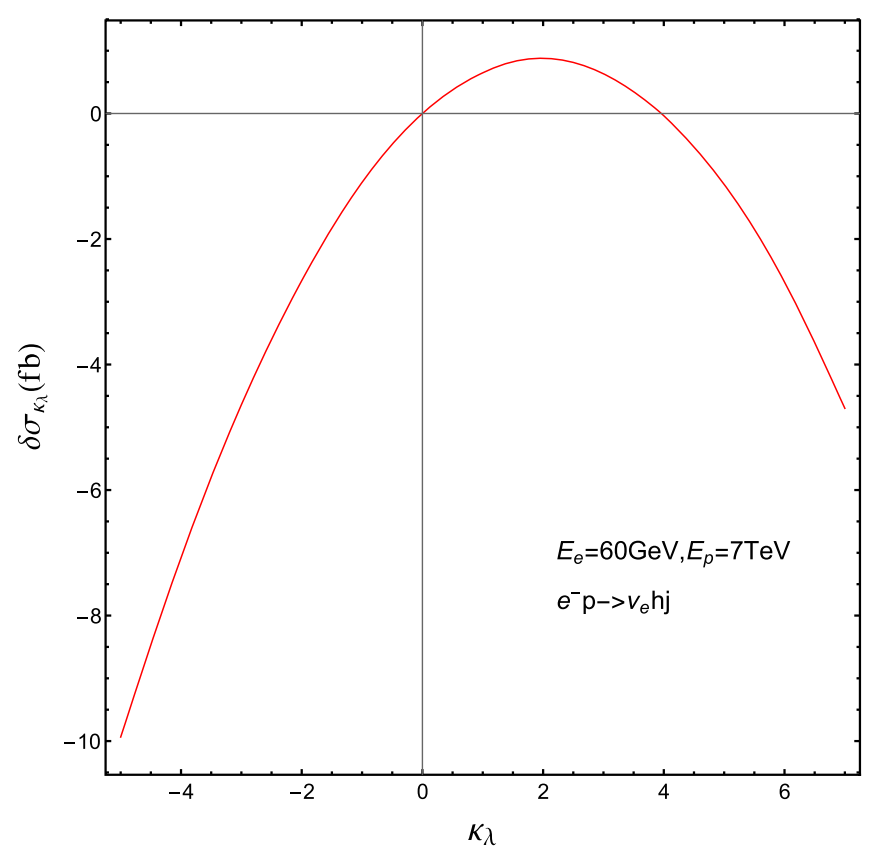

FIG. 5. The cross section $\delta \sigma_{\kappa_{\lambda}}$ from the $\kappa_{\lambda}$ dependent one-loop corrections varying with $\kappa_{\lambda}$, with basic cuts given in Eq. (11). The electron polarization is taken to be $-80 \%$. 


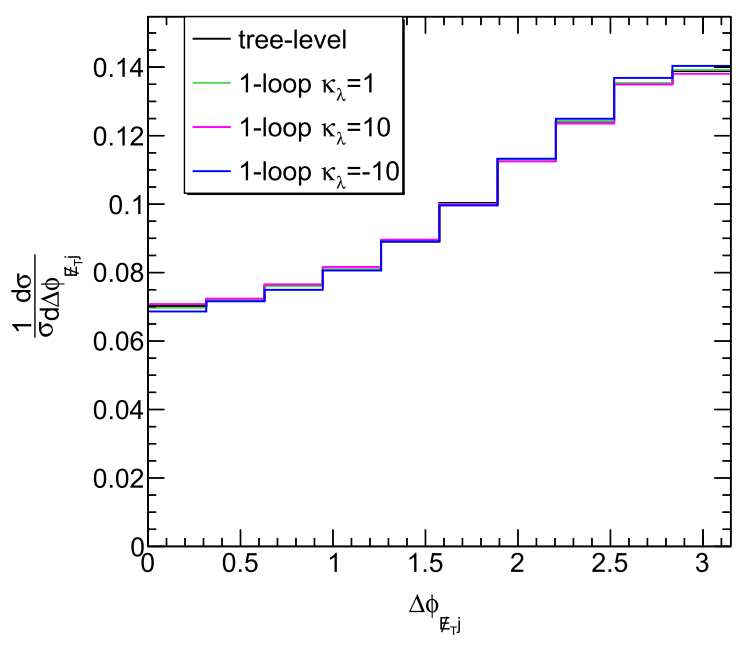

(a) The azimuthal angle $\Delta \phi_{\mathbb{E}_{T} j}$

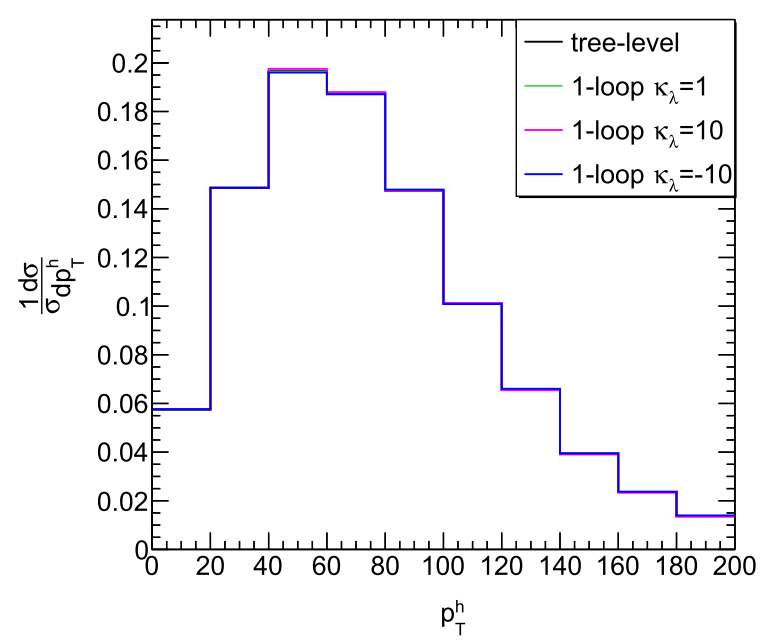

(b) The Higgs transverse momentum $p_{T}^{h}$

FIG. 6. The normalized $\Delta \phi_{\mathscr{E}_{T} j}$ (left panel) and $p_{T}^{h}$ (right panel) distributions with various $\kappa_{\lambda}$.

is absent in the case of loop corrections with the Higgs trilinear self-coupling [51]. This is very well illustrated in Fig. 6 even when $\kappa_{\lambda}$ is varied in a very wide range. The distributions are normalized to reflect only the difference in shape.

As there is little difference in shapes of distributions, we seek to identify the anomalous Higgs self-interaction from SM processes using their production rate. This can be done with the $\chi^{2}$ method, in which the deviation between BSM and SM cross sections are described by

$$
\chi^{2}=\left(\frac{N_{\kappa_{\lambda} \neq 1}-N_{\kappa_{\lambda}=1}}{\sqrt{N_{\kappa_{\lambda}=1}}}\right)^{2}
$$

where $N_{\kappa_{\lambda}=1}$ is the number of events of the $e^{-} p \rightarrow \nu_{e} h j$ with $\kappa_{\lambda}=1$, while $N_{\kappa_{\lambda} \neq 1}$ contains the anomalous $\kappa_{\lambda}$ contribution. The universal contribution from the top and bottom quarks is also included. Since the decay width of Higgs is very small, the number of events can be calculated using the narrow-width approximation

$$
N_{\kappa_{\lambda}}=\mathcal{L} \cdot \sigma_{\kappa_{\lambda}} \cdot \mathrm{BR}_{\kappa_{\lambda}}
$$

where $\mathcal{L}$ is the integrated luminosity, BR is the branch ratio. The dependence of branch ratio on $\kappa_{\lambda}$ has been studied in [49]. We apply the $\chi^{2}$ method to the $H \rightarrow b \bar{b}$ decay channel for its large branch ratio $(\mathrm{BR}(h \rightarrow b \bar{b}) \approx 58 \%)$ [71]. The limits on $\kappa_{\lambda}$ at $95 \%$ C.L. are listed in Table I. We find that $\kappa_{\lambda}$

TABLE I. The 95\% C.L. bounds on $\kappa_{\lambda}$ at parton level for various integrated luminosities.

\begin{tabular}{lc}
\hline \hline The integrated luminosity & Bounds of the $\kappa_{\lambda}$ \\
\hline $\mathcal{L}=1 \mathrm{ab}^{-1}$ & {$[-0.88,3.33]$} \\
$\mathcal{L}=2 \mathrm{ab}^{-1}$ & {$[-0.57,2.98]$} \\
$\mathcal{L}=3 \mathrm{ab}^{-1}$ & {$[-0.42,2.79]$} \\
\hline \hline
\end{tabular}

is better constrained with the increase of the integrated luminosity. The most stringent limits on $\kappa_{\lambda}$ is $[-0.42,2.79]$, with $\mathcal{L}=3 \mathrm{ab}^{-1}$.

The above results are obtained at parton-level without considering any background. Fortunately, deep inelastic scattering machines, e.g., $\mathrm{LHeC}$, and FCC-eh have great potential for high precision Higgs physics. The measurement of $H \rightarrow b \bar{b}$ has reached a precision of $\mathcal{O}(1 \%)$ [72,73], which is good enough for probing the Higgs self-coupling at NLO. References $[59,74]$ have studied the single Higgs production and its background in detail. In [74], both cut based and BDT based analyses are pursued, and reasonable detector setups for $\mathrm{LHeC}$ are considered. For single Higgs production at the $\mathrm{LHeC}$ via $\mathrm{W}$ boson fusion, by imposing appropriate cuts, [74] shows that for $\mathcal{L}=1 \mathrm{ab}^{-1}$, there are about 3600 signal events surviving the cuts with the ratio of the surviving signal

TABLE II. The $95 \%$ C.L. bounds on $\kappa_{\lambda}$ for various integrated luminosities after the cut-based analysis with the signal survival rate being $4.3 \%$.

\begin{tabular}{lc}
\hline \hline The integrated luminosity & Bounds of the $\kappa_{\lambda}$ \\
\hline $\mathcal{L}=1 \mathrm{ab}^{-1}$ & {$[-3.67,6.22]$} \\
$\mathcal{L}=2 \mathrm{ab}^{-1}$ & {$[-2.89,5.43]$} \\
$\mathcal{L}=3 \mathrm{ab}^{-1}$ & {$[-2.49,5.02]$} \\
\hline \hline
\end{tabular}

TABLE III. The $95 \%$ C.L. bounds on $\kappa_{\lambda}$ for various integrated luminosities, assuming the signal survival rate $\epsilon_{S}$ to be $10 \%, 25 \%$ respectively.

\begin{tabular}{lcc}
\hline \hline The integrated luminosity & $\epsilon_{S}=10 \%$ & $\epsilon_{S}=25 \%$ \\
\hline $\mathcal{L}=1 \mathrm{ab}^{-1}$ & {$[-2.74,5.28]$} & {$[-1.97,4.45]$} \\
$\mathcal{L}=2 \mathrm{ab}^{-1}$ & {$[-2.11,4.63]$} & {$[-1.47,3.93]$} \\
$\mathcal{L}=3 \mathrm{ab}^{-1}$ & {$[-1.79,4.30]$} & {$[-1.21,3.67]$} \\
\hline \hline
\end{tabular}




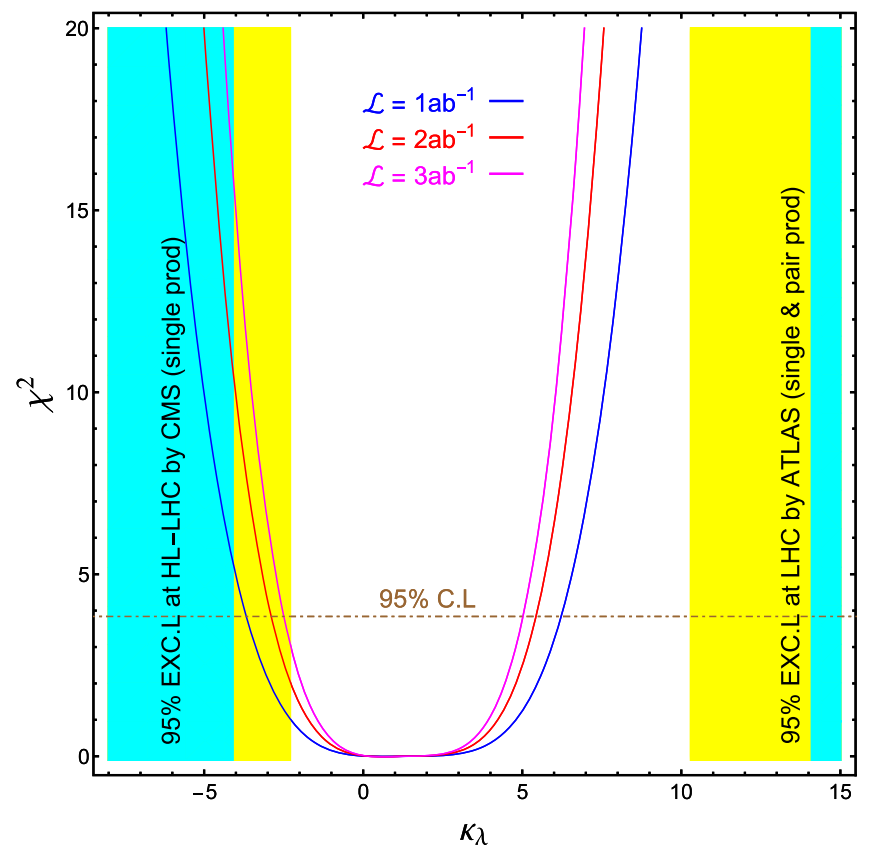

FIG. 7. The $95 \%$ C.L. bounds on $\kappa_{\lambda}$ at the $\mathrm{LHeC}$ for various integrated luminosities, assuming the signal survival rate to be $4.3 \%$. We also plot the $95 \%$ C.L. exclusion of ATLAS, combining single Higgs production and Higgs pair production (cyan shade), and HL-LHC with single Higgs production only (yellow shade).

to background being as large as 2.9. It is also shown that 5 times more signal events will be kept with the BDT method. We adopt the cut based analysis, imposing the same cuts and assuming the same ratio of the surviving signal to background. The survival rate of the signal at $\kappa_{\lambda}=1$ is found to be $4.3 \%$ at the detector level. The bounds on $\kappa_{\lambda}$ in Table I are then broadened to the values shown in Table II. Since more sophisticated methods, such as BDT, will raise the survival rate of signals, we also show the bounds with survival rate of events being 10\%, 25\% respectively in Table III, keeping the ratio of surviving signal to background unchanged. (The 1loop QCD correction to VBF processes at percent level [75] is not included, which has negligible effect on our result.)

The bounds given by cut-based analysis are also shown in Fig. 7, in which the solid blue, red and magenta curves correspond to different integrated luminosities, the cyan shade represents the projection of $95 \%$ C.L. exclusion at the HL-LHC with $3 \mathrm{ab}^{-1}$ by CMS via single Higgs production [30], and the yellow shade represents the recent 95\% C.L. exclusion from combination of double and single Higgs production by ATLAS [57]. The signal exclusion at the $\mathrm{LHeC}$ is clearly more efficient in the $\kappa_{\lambda}>0$ region. However, it should be noted that the analysis of double Higgs production at the HL-LHC gives a better prospective constraint [0.1,2.3] [30]. Our results can be improved if the background in the measurement could be further reduced with more sophisticated methods (e.g., the machine learning, which is helpful in heavy flavor tagging [73]).

\section{CONCLUSION}

In this paper, we study the significance of the Higgs trilinear coupling through the single Higgs production process $e^{-} p \rightarrow \nu_{e} h j$ at the $\mathrm{LHeC}$. The analytical calculation is carried out up to one loop level for both $\lambda_{3}$ dependent and independent intermediate states. The analytical results are then used in the Monte Carlo simulation to produce numerically the cross section at various $\kappa_{\lambda}$, which allows us to quantify the deviation of the cross section from the SM case $\left(\kappa_{\lambda}=1\right)$ in a $\chi^{2}$ statistic analysis. From this analysis we find that the 95\% C.L. bound for $\kappa_{\lambda}$ is $[-2.89,5.43]([-2.11,4.63])$ with a $2 \mathrm{ab}^{-1}$ integrated luminosity, after using the result from an existing cut-based analysis, assuming the survival rate of signal being $4.3 \%$ $(10 \%)$. This is a significant improvement compared with the current experimental result in the $\kappa_{\lambda}>0$ region. We expect the result to be improved with more accurate measurement of Higgs decays.

\section{ACKNOWLEDGMENTS}

We thank Dr. Chen Shen, Prof. Uta Klein and LHeC Higgs \& Top group for helpful discussion. The work is supported in part by the National Science Foundation of China (11875232) and the Zhejiang University Fundamental Research Funds for the Central Universities. KW is also supported by Zhejiang University K.P Chao High Technology Development Foundation.
[1] ATLAS Collaboration, Observation of a new particle in the search for the Standard Model Higgs boson with the ATLAS detector at the LHC, Phys. Lett. B 716, 1 (2012).

[2] CMS Collaboration, Observation of a new boson at a mass of $125 \mathrm{GeV}$ with the CMS experiment at the LHC, Phys. Lett. B 716, 30 (2012).
[3] ATLAS and CMS Collaborations, Combined Measurement of the Higgs Boson Mass in $p p$ Collisions at $\sqrt{s}=7$ and $8 \mathrm{TeV}$ with the ATLAS and CMS Experiments, Phys. Rev. Lett. 114, 191803 (2015).

[4] CMS Collaboration, Constraints on the spin-parity and anomalous HVV couplings of the Higgs boson in proton collisions at 7 and 8 TeV, Phys. Rev. D 92, 012004 (2015). 
[5] ATLAS Collaboration, Search for a $C P$-odd Higgs boson decaying to $\mathrm{Zh}$ in $\mathrm{pp}$ collisions at $\sqrt{s}=8 \mathrm{TeV}$ with the ATLAS detector, Phys. Lett. B 744, 163 (2015).

[6] ATLAS and CMS Collaborations, Measurements of the Higgs boson production and decay rates and constraints on its couplings from a combined ATLAS and CMS analysis of the LHC pp collision data at $\sqrt{s}=7$ and $8 \mathrm{TeV}$, J. High Energy Phys. 08 (2016) 045.

[7] ATLAS Collaboration, Cross-section measurements of the Higgs boson decaying into a pair of $\tau$-leptons in protonproton collisions at $\sqrt{s}=13 \mathrm{TeV}$ with the ATLAS detector, Phys. Rev. D 99, 072001 (2019).

[8] ATLAS Collaboration, Observation of $H \rightarrow b \bar{b}$ decays and $V H$ production with the ATLAS detector, Phys. Lett. B 786, 59 (2018).

[9] A. Noble and M. Perelstein, Higgs self-coupling as a probe of electroweak phase transition, Phys. Rev. D 78, 063518 (2008).

[10] M. Trodden, Electroweak baryogenesis, Rev. Mod. Phys. 71, 1463 (1999).

[11] D. E. Morrissey and M. J. Ramsey-Musolf, Electroweak baryogenesis, New J. Phys. 14, 125003 (2012).

[12] CMS Collaboration, Search for Higgs boson pair production in the $\gamma \gamma \mathrm{b} \bar{b}$ final state in pp collisions at $\sqrt{s}=13 \mathrm{TeV}$, Phys. Lett. B 788, 7 (2019).

[13] ATLAS Collaboration, Search for Higgs boson pair production in the $\gamma \gamma b \bar{b}$ final state with $13 \mathrm{TeV} p p$ collision data collected by the ATLAS experiment, J. High Energy Phys. 11 (2018) 040.

[14] ATLAS Collaboration, Search for pair production of Higgs bosons in the $b \bar{b} b \bar{b}$ final state using proton-proton collisions at $\sqrt{s}=13 \mathrm{TeV}$ with the ATLAS detector, J. High Energy Phys. 01 (2019) 030.

[15] CMS Collaboration, Combination of Searches for Higgs Boson Pair Production in Proton-Proton Collisions at $\sqrt{s}=13$ TeV, Phys. Rev. Lett. 122, 121803 (2019).

[16] ATLAS Collaboration, Combination of searches for Higgs boson pairs in $p p$ collisions at $\sqrt{s}=13 \mathrm{TeV}$ with the ATLAS detector, Phys. Lett. B 800, 135103 (2020).

[17] U. Baur, T. Plehn, and D. L. Rainwater, Measuring the Higgs Boson Self Coupling at the LHC and Finite Top Mass Matrix Elements, Phys. Rev. Lett. 89, 151801 (2002).

[18] U. Baur, T. Plehn, and D. L. Rainwater, Determining the Higgs boson self-coupling at hadron colliders, Phys. Rev. D 67, 033003 (2003).

[19] U. Baur, T. Plehn, and D. L. Rainwater, Probing the Higgs self-coupling at hadron colliders using rare decays, Phys. Rev. D 69, 053004 (2004).

[20] M. Moretti, S. Moretti, F. Piccinini, R. Pittau, and A. D. Polosa, Higgs boson self-couplings at the LHC as a probe of extended Higgs sectors, J. High Energy Phys. 02 (2005) 024.

[21] M. J. Dolan, C. Englert, and M. Spannowsky, Higgs selfcoupling measurements at the LHC, J. High Energy Phys. 10 (2012) 112.

[22] J. Baglio, A. Djouadi, R. Gröber, M. M. Mühlleitner, J. Quevillon, and M. Spira, The measurement of the Higgs self-coupling at the LHC: Theoretical status, J. High Energy Phys. 04 (2013) 151.
[23] F. Goertz, A. Papaefstathiou, L. L. Yang, and J. Zurita, Measuring the Higgs boson self-coupling at the LHC using ratios of cross sections, in 25th Rencontres de Blois on Particle Physics and Cosmology Blois, France, 2013 (2013) [arXiv:1309.3805].

[24] R. Frederix, S. Frixione, V. Hirschi, F. Maltoni, O. Mattelaer, P. Torrielli, E. Vryonidou, and M. Zaro, Higgs pair production at the LHC with NLO and parton-shower effects, Phys. Lett. B 732, 142 (2014).

[25] Q.-H. Cao, Y. Liu, and B. Yan, Measuring trilinear Higgs coupling in $\mathrm{WHH}$ and $\mathrm{ZHH}$ productions at the highluminosity LHC, Phys. Rev. D 95, 073006 (2017).

[26] M. Gouzevitch, A. Oliveira, J. Rojo, R. Rosenfeld, G. P. Salam, and V. Sanz, Scale-invariant resonance tagging in multijet events and new physics in Higgs pair production, J. High Energy Phys. 07 (2013) 148.

[27] J. K. Behr, D. Bortoletto, J. A. Frost, N. P. Hartland, C. Issever, and J. Rojo, Boosting Higgs pair production in the $b \bar{b} b \bar{b}$ final state with multivariate techniques, Eur. Phys. J. C 76, 386 (2016).

[28] F. Bishara, R. Contino, and J. Rojo, Higgs pair production in vector-boson fusion at the LHC and beyond, Eur. Phys. J. C 77, 481 (2017).

[29] S. Di Vita, C. Grojean, G. Panico, M. Riembau, and T. Vantalon, A global view on the Higgs self-coupling, J. High Energy Phys. 09 (2017) 069.

[30] HL/HE WG2 Group Collaborations, Higgs Physics at the HL-LHC and HE-LHC, arXiv:1902.00134.

[31] H. Baer, T. Barklow, K. Fujii, Y. Gao, A. Hoang, S. Kanemura et al., The International Linear Collider Technical Design Report-Volume 2: Physics, arXiv:1306.6352.

[32] D. M. Asner et al., ILC Higgs White Paper, in Proceedings, 2013 Community Summer Study on the Future of U.S. Particle Physics: Snowmass on the Mississippi (CSS2013): Minneapolis, MN, USA, 2013 (2013) [arXiv:1310.0763].

[33] S. Di Vita, G. Durieux, C. Grojean, J. Gu, Z. Liu, G. Panico, M. Riembau, and T. Vantalon, A global view on the Higgs self-coupling at lepton colliders, J. High Energy Phys. 02 (2018) 178.

[34] F. Maltoni, D. Pagani, and X. Zhao, Constraining the Higgs self-couplings at $e^{+} e^{-}$colliders, J. High Energy Phys. 07 (2018) 087.

[35] W. Yao, Studies of measuring Higgs self-coupling with $H H \rightarrow b \bar{b} \gamma \gamma$ at the future hadron colliders, in Proceedings, 2013 Community Summer Study on the Future of U.S. Particle Physics: Snowmass on the Mississippi (CSS2013): Minneapolis, MN, USA, 2013 (2013). http://www.slac .stanford.edu/econf/C1307292/docs/submittedArxivFiles/ 1308.6302.pdf.

[36] A. J. Barr, M. J. Dolan, C. Englert, D. E. Ferreira de Lima, and M. Spannowsky, Higgs self-coupling measurements at a $100 \mathrm{TeV}$ hadron collider, J. High Energy Phys. 02 (2015) 016.

[37] A. Azatov, R. Contino, G. Panico, and M. Son, Effective field theory analysis of double Higgs boson production via gluon fusion, Phys. Rev. D 92, 035001 (2015).

[38] H.-J. He, J. Ren, and W. Yao, Probing new physics of cubic Higgs boson interaction via Higgs pair production at hadron colliders, Phys. Rev. D 93, 015003 (2016). 
[39] C.-Y. Chen, Q.-S. Yan, X. Zhao, Y.-M. Zhong, and Z. Zhao, Probing triple-Higgs productions via $4 b 2 \gamma$ decay channel at a $100 \mathrm{TeV}$ hadron collider, Phys. Rev. D 93, 013007 (2016).

[40] R. Contino et al., Physics at a $100 \mathrm{TeV}$ pp collider: Higgs and EW symmetry breaking studies, CERN Yellow Rep. 255 (2017).

[41] S. Banerjee, C. Englert, M. L. Mangano, M. Selvaggi, and M. Spannowsky, $h h+$ jet production at $100 \mathrm{TeV}$, Eur. Phys. J. C 78, 322 (2018).

[42] J. Chang, K. Cheung, J. S. Lee, C.-T. Lu, and J. Park, Higgsboson-pair production $H(\rightarrow b \bar{b}) H(\rightarrow \gamma \gamma)$ from gluon fusion at the HL-LHC and HL-100 TeV hadron collider, Phys. Rev. D 100, 096001 (2019).

[43] A. Blondel and P. Janot, Future strategies for the discovery and the precise measurement of the Higgs self-coupling, arXiv:1809.10041.

[44] J. H. Kim, Y. Sakaki, and M. Son, Combined analysis of double Higgs production via gluon fusion at the HL-LHC in the effective field theory approach, Phys. Rev. D 98, 015016 (2018).

[45] J. H. Kim, K. Kong, K. T. Matchev, and M. Park, Probing the Triple Higgs Self-Interaction at the Large Hadron Collider, Phys. Rev. Lett. 122, 091801 (2019).

[46] J. H. Kim, M. Kim, K. Kong, K. T. Matchev, and M. Park, Portraying double Higgs at the large Hadron collider, J. High Energy Phys. 09 (2019) 047.

[47] M. McCullough, An indirect model-dependent probe of the Higgs self-coupling, Phys. Rev. D 90, 015001 (2014).

[48] C. Shen and S.-h. Zhu, Anomalous Higgs-top coupling pollution of the triple Higgs coupling extraction at a future high-luminosity electron-positron collider, Phys. Rev. D 92, 094001 (2015).

[49] G. Degrassi, P. P. Giardino, F. Maltoni, and D. Pagani, Probing the Higgs self-coupling via single Higgs production at the LHC, J. High Energy Phys. 12 (2016) 080.

[50] W. Bizon, M. Gorbahn, U. Haisch, and G. Zanderighi, Constraints on the trilinear Higgs coupling from vector boson fusion and associated Higgs production at the LHC, J. High Energy Phys. 07 (2017) 083.

[51] F. Maltoni, D. Pagani, A. Shivaji, and X. Zhao, Trilinear Higgs coupling determination via single-Higgs differential measurements at the LHC, Eur. Phys. J. C 77, 887 (2017).

[52] J. Fleischer and F. Jegerlehner, Radiative corrections to Higgs production by $e^{+} e^{-} \rightarrow Z H$ in the Weinberg-Salam model, Nucl. Phys. B216, 469 (1983).

[53] A. Denner, J. Kublbeck, R. Mertig, and M. Bohm, Electroweak radiative corrections to $e+e-\rightarrow H Z$, Z. Phys. C 56, 261 (1992).

[54] A. Denner, S. Dittmaier, M. Roth, and M. M. Weber, Electroweak radiative corrections to $e+e-\rightarrow \nu \bar{\nu} H$, Nucl. Phys. B660, 289 (2003).

[55] G. Belanger, F. Boudjema, J. Fujimoto, T. Ishikawa, T. Kaneko, K. Kato, and Y. Shimizu, Full one loop electroweak radiative corrections to single Higgs production in e+ e-, Phys. Lett. B 559, 252 (2003).

[56] ATLAS Collaboration, Constraint of the Higgs Boson SelfCoupling from Higgs Boson Differential Production and
Decay Measurements, CERN Report No. ATL-PHYS-PUB2019-009, 2019.

[57] ATLAS Collaboration, Constraints on the Higgs Boson Self-Coupling from the Combination of Single-Higgs and Double-Higgs Production Analyses Performed with the ATLAS Experiment, CERN Report No. ATLAS-CONF2019-049, 2019.

[58] LHeC Study Group Collaboration, A large hadron Electron collider at CERN: Report on the physics and design concepts for machine and detector, J. Phys. G 39, 075001 (2012).

[59] T. Han and B. Mellado, Higgs Boson Searches and the H b anti-b Coupling at the LHeC, Phys. Rev. D 82, 016009 (2010).

[60] M. Kumar, X. Ruan, R. Islam, A. S. Cornell, M. Klein, U. Klein, and B. Mellado, Probing anomalous couplings using di-Higgs production in electron-proton collisions, Phys. Lett. B 764, 247 (2017).

[61] M. Kumar, X. Ruan, A. S. Cornell, R. Islam, and B. Mellado, Double Higgs production at FCC-he and prospects for measurements of self-coupling, J. Phys. Conf. Ser. 623, 012017 (2015).

[62] A. Efrati and Y. Nir, What if $\lambda_{h h h} \neq 3 m_{h}^{2} / v$, arXiv:1401 .0935 .

[63] T. Hahn, Generating Feynman diagrams and amplitudes with FeynArts 3, Comput. Phys. Commun. 140, 418 (2001).

[64] T. Hahn and M. Perez-Victoria, Automatized one loop calculations in four-dimensions and D-dimensions, Comput. Phys. Commun. 118, 153 (1999).

[65] A. Denner, Techniques for calculation of electroweak radiative corrections at the one loop level and results for $\mathrm{W}$ physics at LEP-200, Fortschr. Phys. 41, 307 (1993).

[66] R. Mertig, M. Bohm, and A. Denner, FEYN CALC: Computer algebraic calculation of Feynman amplitudes, Comput. Phys. Commun. 64, 345 (1991).

[67] V. Shtabovenko, R. Mertig, and F. Orellana, New developments in FeynCalc 9.0, Comput. Phys. Commun. 207, 432 (2016).

[68] T. Hahn, CUBA: A library for multidimensional numerical integration, Comput. Phys. Commun. 168, 78 (2005).

[69] C. Schmidt, J. Pumplin, D. Stump, and C. P. Yuan, CT14QED parton distribution functions from isolated photon production in deep inelastic scattering, Phys. Rev. D 93, 114015 (2016).

[70] J. Alwall, R. Frederix, S. Frixione, V. Hirschi, F. Maltoni, O. Mattelaer, H.-S. Shao, T. Stelzer, P. Torrielli, and M. Zaro, The automated computation of tree-level and next-toleading order differential cross sections, and their matching to parton shower simulations, J. High Energy Phys. 07 (2014) 079.

[71] M. Tanabashi et al. Particle Data Group Collaboration, Review of particle physics, Phys. Rev. D 98, 030001 (2018).

[72] M. Klein, Future deep inelastic scattering with the LHeC, in From My Vast Repertoire: Guido Altarelli's Legacy, edited by A. Levy, S. Forte, and G. Ridolfi (2019), pp. 303-347 [arXiv:1802.04317]. 
[73] FCC Collaboration, FCC physics opportunities, Eur. Phys. J. C 79, 474 (2019).

[74] T. Mashiro, SM Higgs at the LHeC, in Proceedings, 25th International Workshop on Deep-Inelastic Scattering and Related Topics (DIS 2017): Birmingham, UK, 2017 (2017), https://indico.cern.ch/event/568360/contributions/2523555/
attachments/1440097/2216668/mtanaka_DIS2017_SMhiggs_ final.pdf.

[75] B. Jager, Next-to-leading order QCD corrections to Higgs production at a future lepton-proton collider, Phys. Rev. D 81, 054018 (2010). 